\title{
Insulin kinetics and the Neonatal Intensive Care Insulin-Nutrition-Glucose (NICING) model
}

$$
\text { JL Dickson }{ }^{a^{*}} \text {, CG Pretty }{ }^{\mathrm{a}}, \mathrm{J} \text { Alsweiler }{ }^{\mathrm{b}} \text {, A. Lynn }{ }^{\mathrm{c}}, \text { JG Chase }^{\mathrm{a}}
$$

${ }^{a}$ Department of Mechanical Engineering, University of Canterbury, Christchurch, New Zealand

${ }^{\mathrm{b}}$ Department of Paediatrics: Child and Youth Health, Auckland, New Zealand and Liggins Institute, University of Auckland, Auckland, New Zealand

'Christchurch Women's’ Hospital Neonatal Intensive Care Unit, Christchurch, New Zealand

* Corresponding author: Jennifer Dickson

Address:

Jennifer Dickson

Department of Mechanical Engineering

University of Canterbury

Private Bag 4800

Christchurch 8140

New Zealand

email: jennifer.dickson@canterbury.ac.nz

Phone: +64 33642987 exnt. 7650

\begin{tabular}{|l|l|l|}
\hline Name & First Affiliation & Email \\
\hline $\begin{array}{l}\text { Dr. Jennifer } \\
\text { Dickson }\end{array}$ & $\begin{array}{l}\text { University of Canterbury, New } \\
\text { Zealand }\end{array}$ & jennifer.dickson@ canterbury.ac.nz \\
\hline $\begin{array}{l}\text { Dr. Christopher } \\
\text { Pretty }\end{array}$ & $\begin{array}{l}\text { University of Canterbury, New } \\
\text { Zealand }\end{array}$ & chris.pretty@ canterbury.ac.nz \\
\hline $\begin{array}{l}\text { Dr. Jane } \\
\text { Alsweiler }\end{array}$ & University of Auckland, New Zealand & j.alsweiler@ auckland.ac.nz \\
\hline $\begin{array}{l}\text { Dr. Adrienne } \\
\text { Lynn }\end{array}$ & Christchurch Women's Hospital & Adrienne.Lynn@ cdhb.health.nz \\
\hline $\begin{array}{l}\text { Prof. J. Geoffrey } \\
\text { Chase }\end{array}$ & $\begin{array}{l}\text { University of Canterbury, New } \\
\text { Zealand }\end{array}$ & geoff.chase@ canterbury.ac.nz \\
\hline
\end{tabular}




\begin{abstract}
Background: Models of human glucose-insulin physiology have been developed for a range of uses, with similarly different levels of complexity and accuracy. STAR (Stochastic Targeted) is a modelbased approach to glycaemic control. Elevated blood glucose concentrations (hyperglycaemia) are a common complication of stress and prematurity in very premature infants, and have been associated with worsened outcomes and higher mortality. This research identifies and validates the model parameters for model-based glycaemic control in neonatal intensive care.
\end{abstract}

Methods: C-peptide, plasma insulin, and BG from a cohort of 41 extremely pre-term (median age 27.2 [26.2 - 28.7] weeks) and very low birth weight infants (median birth weight 839 [735 - 1000] g) are used alongside C-peptide kinetic models to identify model parameters associated with insulin kinetics in the NICING (neonatal intensive care insulin-nutrition-glucose) model. A literature analysis is used to determine models of kidney clearance and body fluid compartment volumes. The full, final NICING model is validated by fitting the model to a cohort of 160 glucose, insulin, and nutrition data records from extremely premature infants from two different NICUs (neonatal intensive care units).

Results: 6 Model parameters related to insulin kinetics were identified. The resulting NICING model is more physiologically descriptive than prior model iterations, including clearance pathways of insulin via the liver and kidney, rather than a lumped parameter. In addition, insulin diffusion between plasma and interstitial spaces is evaluated, with differences in distribution volume taken into consideration for each of these spaces. The NICING model was shown to fit clinical data well, with a low model fit error similar to that of previous model iterations.

Conclusions: Insulin kinetic parameters have been identified, and the NICING model is presented for glycaemic control neonatal intensive care. The resulting NICING model is more complex and physiologically relevant, with no loss in bedside-identifiability or ability to capture and predict metabolic dynamics.

Keywords: physiological modelling, glucose, insulin, premature infant, glycaemic control 


\section{Introduction}

Mathematical models of glucose-insulin physiology have been developed with differing levels of complexity for a wide range of scientific and clinical applications. Models developed for the determination of model-based measures of physiology (e.g. [1, 2]), such as insulin sensitivity, tend to be more complex and comprehensive, requiring higher data density and/or measurement of multiple metabolic species. Other models are designed for specific clinical applications, such as glycaemic control in intensive care (e.g. [3-6]), or Type 1 Diabetes cohorts (e.g.[7]). These models tend to be less complex, as metabolic measurements are minimised in clinical or outpatient settings due to clinical and patient factors such as cost, availability, or comfort. In general, physiological models must have appropriate resolution, be mathematically identifiable [8], as well as practically applicable within their chosen application [9].

While glucose-insulin models for adult intensive care applications are more widely documented [9, 10] virtually no work has looked at neonatal intensive care unit (NICU) applications. Elevated blood glucose levels (BG) (Hyperglycaemia) is a common complication of prematurity and stress in neonatal intensive care, and while definitions and thresholds vary [11], studies show that $30-70 \%$ of very/extremely low birth weight infants have at least one BG> $8 \mathrm{mmol} / \mathrm{L}$ [12-16]. Hyperglycaemia is associated with increased mortality [15-17], and morbidity/complications in this cohort [15-21], but there is still debate over whether hyperglycaemia causes increased morbidity, or is reflective of worsened condition.

There is no best practice method for the treatment of hyperglycaemia in this cohort. Use of insulin has been shown to increase glucose tolerance [22-27], resulting in increased weight gain [23, 25, 27], but also commonly results in increased incidence of hypoglycaemia (low blood glucose concentration) [28-30], which is also dangerous. In adult intensive care even a single hypoglycaemic episode has been associated with increased risk of mortality [31-33], while in neonatal intensive care hypoglycaemia as been associated with adverse neurological outcomes [34, 35]. Model-based methods for glycaemic control have been little investigated, due in part to the extremely fragile nature 
of this cohort and the subsequent limitations on invasive procedures and blood sample collection [36] that thus also limit the ability to identify parameters to validate more physiologically relevant and complex models.

The aim of this study is twofold. First, this paper presents a clinically applicable and physiologically relevant model of glucose-insulin physiology in hyperglycaemic very/extremely premature infants (gestational age $<32$ weeks), and secondly it aims to identify insulin kinetic parameters for this model. With regards to the first aim, it combines a previous, simpler, model iteration in this cohort [37] with a more physiologically descriptive model currently utilised for glycaemic control in the adult intensive care unit [5]. Specifically, this model is more descriptive with regards to modelled insulin kinetics and dynamics [38], necessary for safe and effective glycaemic control.

Related to the second aim, this paper also focuses on parameter identification of modelled insulin kinetics. While the key glucose dynamics have been previously published [39, 40], this study uses a previously published methodology from adults [41] and a novel data set of C-peptide concentrations from a very low birth weight $(<1500 \mathrm{~g})$ cohort to evaluate diffusion of insulin between plasma and the interstitial fluid, and total liver and kidney clearance of insulin. In addition, the assumptions around insulin distribution volumes in the plasma and interstitial compartments are examined, and kidney glomerular filtration rate (GFR) is used to provide a patient specific value for renal clearance of insulin. The revised kinetics model is then used to create a new more physiologically relevant and complex, yet equally identifiable, glucose-insulin model, which is validated using clinical data. 


\subsection{Models and Methods}

\subsection{NICING Model of Glucose-Insulin Physiology}

The NICING (Neonatal Intensive Care Insulin-Nutrition-Glucose) model for glycaemic control in very/extremely preterm neonates is developed from a previous NICU model [37] and the ICING (Intensive Care Insulin-Nutrition-Glucose) model for adult intensive care [5]. The values given, with the exception of those derived in this study, are predominantly derived from literature, and are originally presented and discussed in [37, 39].

In the new NICING model, blood glucose $(G \in \mathbb{R}: G \geq 0)$, plasma $(I \in \mathbb{R}: I \geq 0)$ and peripheral $(Q \in \mathbb{R}: Q \geq 0)$ insulin kinetics are described:

$$
\begin{gathered}
\dot{G}=-p_{G} G(t)-S_{I} G(t) \frac{Q(t)}{1+\alpha_{G} Q(t)}+\frac{P_{e x}(t)+E G P * m_{\text {body }}-C N S * m_{\text {brain }}}{V_{g, \text { frac }}(t) * m_{\text {body }}} \\
\dot{I}=-\frac{n_{L} I(t)}{1+\alpha_{I} I(t)}-n_{K} I(t)-n_{I}(I(t)-Q(t))+\frac{u_{e x}(t)}{V_{P} * m_{\text {body }}}+\left(1-x_{L}\right) u_{e n} \\
\dot{Q}=n_{I} \frac{V_{P}}{V_{Q}}(I(t)-Q(t))-n_{C} \frac{Q(t)}{1+\alpha_{G} Q(t)}
\end{gathered}
$$

Where $G$ has units of $\mathrm{mmol} / \mathrm{L} / \mathrm{min}$, and $I$ and $Q$ have units of $\mathrm{mU} / \mathrm{L} / \mathrm{min}$. Clearance of glucose includes both insulin mediated and non-insulin mediated routes. Insulin mediated uptake is modulated by insulin sensitivity $\left(s_{I} \in \mathbb{R}: s_{I} \geq 10^{-7}\right.$ ), while non-insulin mediated routes include a brain-mass ( $m_{\text {brain }} \sim 14 \%$ whole body mass, $m_{\text {body }}$ ) dependant $[40]$ central nervous system uptake $(\mathrm{CNS}=0.088$ $\mathrm{mmol} / \mathrm{kg} / \mathrm{min}$ ), and a concentration dependant pathway capturing other glucose clearances such as from the kidney $\left(p_{G}=0.003 / \mathrm{min}\right)$. As $p_{G}$ trades off mathematically with $S_{I}$ and cannot be directly measured in this cohort, the adult value of $0.003 / \mathrm{min}$ is assumed. Glucose enters the system via exogenous $\left(P_{e x}\right)$ inputs (parenteral and enteral) and endogenous glucose production $(E G P=0.033$ $\mathrm{mmol} / \mathrm{kg} / \mathrm{min}$ ) by the liver [39]. $V_{g, f r a c}$ is the volume of distribution of glucose in plasma in litres and is based on gestational age [37]. Saturation of insulin mediated glucose uptake in adults is modulated 
with a Michaelis-Menten function, characterised by the parameter $\alpha_{G}$. For neonates no saturation has been observed [42], so this value is $\alpha_{G}=0$.

Liver clearance of insulin occurs in two main processes, a first pass hepatic clearance of endogenously secreted insulin $\left(x_{L}\right)$ and clearance of insulin from circulating blood (rate constant: $n_{L}$ ). This hepatic clearance is a receptor-mediated process resulting in saturation of clearance at high insulin concentrations [43, 44], and so is modelled with a Michaelis-Menten function, characterised by the parameter $\alpha_{I}$. Saturation of liver clearance and first pass hepatic clearance of insulin cannot be measured in premature infants, or indirectly determined in this analysis, so the adult value of $\alpha_{I}=$ $0.0017 \mathrm{~L} / \mathrm{mU}$ and $x_{L}=0.67$ are used $[5,45]$.

Kidney clearance of insulin (rate constant: $n_{K}$ ) includes both glomerular filtration of insulin, and proximal tubal reabsorption. Insulin movement between the plasma and interstitial fluid, (rate constant: $n_{I}$ ) is likely diffusion based, as it is not reported to be saturated [46, 47]. Insulin degradation by cells (rate constant: $n_{C}$ ) is a complex, receptor-mediated process. Receptor bound insulin can either be released back into the extracellular fluid space or internalised by the cell [43]. As insulin-binding and insulin-receptor mediated glucose uptake are related, both share the saturation parameter $\alpha_{G}=0$. Insulin is secreted $\left(u_{e n}\right)$ or is exogenously delivered $\left(u_{e x}\right)$, with units of $\mathrm{mU} / \mathrm{min}$. Insulin secretion can be calculated using C-peptide $[48,49]$, a molecule secreted in equimolar quantities to insulin with simpler clearance kinetics. If C-Peptide measurements are not available, insulin secretion is modelled $[49,50]:$

$$
u_{e n}= \begin{cases}\max (4.2,-1.5+1.9 * G) & \text { if female } \\ \max (2.2,-0.37+0.86 * G) & \text { if male }\end{cases}
$$

The volume of distribution of plasma insulin is assumed to be the blood plasma volume $\left(V_{P}\right)$. The volume of distribution of insulin in the peripheral compartment is approximated as the interstitial fluid volume $\left(V_{Q}\right)$. 


\subsection{C-peptide Kinetic Model}

C-peptide is a protein secreted in equimolar quantities with insulin. However, unlike insulin, it is only cleared by the kidney. Therefore, the relatively simple kinetics of C-peptide provide a means to estimate insulin secretion. The well known 2 compartment kinetics model [48] is used due to its overall physiological relevance:

$$
\begin{gathered}
\frac{d \hat{C}}{d t}=\hat{S}-\left(k_{1}+k_{3}\right) \hat{C}+k_{2} \hat{Y} \\
\frac{d \hat{Y}}{d t}=k_{1} \hat{C}-k_{2} \hat{Y}
\end{gathered}
$$

Where $\hat{C}(\hat{C} \in \mathbb{R}: \hat{C} \geq 0$ [pmol]) is the amount of C-peptide in the central compartment of plasma (and tissues in rapid equilibrium with the plasma), and $\hat{Y}(\hat{Y} \in \mathbb{R}: \hat{Y} \geq 0$ [pmol]) is the amount of Cpeptide in the peripheral extra vascular compartment. $\hat{S}$ is the rate at which C-peptide, and thus insulin, is secreted into the central compartment [pmol/min]. Assuming the same diffusive properties between compartments gives $k_{2}=k_{1}\left(V_{P} / V_{Q}\right)$, and considering the concentrations of C-peptide in central and peripheral compartments $(C, Y \in \mathbb{R}: C, Y \geq 0)$ with units $[\mathrm{pmol} / \mathrm{L}]$, allows the model to be rewritten:

$$
\begin{gathered}
\frac{d C}{d t}=S-n_{I_{c p}}(C-Y)-n_{k_{c p}} C \\
\frac{d Y}{d t}=n_{I_{c p}} \frac{V_{P}}{V_{Q}}(C-Y)
\end{gathered}
$$

Where:

$$
\begin{aligned}
& n_{I_{c p}}=k_{1} \\
& n_{k_{c p}}=k_{3}
\end{aligned}
$$

The rate parameter $n_{I_{c p}}$ describes the rate of transport of $\mathrm{C}$-peptide from the central to the peripheral compartment, and vice versa, and assumes that $k_{2}=k_{1}\left(V_{P} / V_{Q}\right)$, in the model presented in [48]. The parameter $n_{k_{c p}}$ describes irreversible removal of C-peptide from central compartment via the kidney. Rate parameters in Equation 9 are renamed to allow consistency with the NICING model structure, 
and reflect the assumption that the rates of forward and reverse diffusion between compartments are equal.

Since no studies have been performed in preterm or term neonates to determine the C-peptide kinetics, adult data and methodology $[48,51]$ was used as a reasonable approximation since the kinetic compartments and their functional physiology are the same. The values for these parameters are given in Table 1, and were adapted to this cohort using the methods in [48] and an age of 0 years, according to the equation:

$$
\begin{gathered}
\text { long half life }(\min )=0.14 * \text { age }(\text { years })+29 \\
k_{2}=F(b-a)+a \\
k_{3}=\frac{a b}{k_{2}} \\
k_{1}=a+b-k_{2}-k_{3}
\end{gathered}
$$

where $a=\log (2) /($ short half life $)$, and $b=\log (2) /(\operatorname{long}$ half life). This age-based extrapolation from adult data does not utilise the model within the limits of the cohort it was originally validated in. This extrapolation was necessary, as C-peptide kinetic data is not available for the premature neonate. However, all parameters fall within the adult ranges (Van Cauter et al., 1992, Polonsky et al., 1986) and were these parameters to scale differently in neonates, it would simply offset SI without changing underlying dynamics. As such, these values within the adult range are considered a justifiable estimation of neonatal behaviour.

Table 1: C-peptide model parameter values

\begin{tabular}{cc}
\hline Parameter & Value [1/min] \\
\hline $\mathrm{k}_{1}$ & 0.0478 \\
$\mathrm{k}_{2}$ & 0.0516 \\
$\mathrm{k}_{3}$ & 0.0644 \\
\hline
\end{tabular}

Sampling constraints due to limited blood volume in this cohort meant frequent, serial measurements of C-peptide were not possible. Assuming steady state conditions, it follows from Equation 8 that the rate of C-peptide entering and leaving the peripheral compartment must be equal. Hence, substituting this equality into Equation 5 and rearranging yields: 


$$
S=n_{k_{c p}} C=k_{3} C
$$

Since insulin is secreted in equimolar quantities alongside C-peptide, then under steady state conditions the rate of secretion of insulin, $u_{e n}$, can be estimated from the rate of excretion of C-peptide from the central compartment.

\subsection{Insulin Kinetic Parameter Identification}

\subsubsection{Insulin Clearance Parameters}

Insulin clearance kinetics were determined using the model-based method of [41], which used Cpeptide kinetics in combination with the ICING model and patient data to determine appropriate insulin clearance parameter values. The diffusion constant for insulin between the plasma and interstitial compartments is modelled as the C-peptide transfer rate scaled by molar mass [41]:

$$
n_{I}=n_{I C P} \frac{m_{c}}{m_{I}}=k_{1} \frac{m_{c}}{m_{I}}
$$

Where $m_{c}=3.02 \mathrm{kDa}$ and $m_{I}=5.8 \mathrm{kDa}$ are the molecular masses of C-Peptide and Insulin, respectively. Diffusion can be complex to characterise, so sensitivity to this parameter was tested by multiplying the mass ratio by powers of $1 / 3$ to $1 / 1$, with the former power based on Einstein's theory for diffusion of molecules in solution [52].

To determine $n_{C}$, steady state is assumed and Equation 3 re-arranged to yield:

$$
n_{C}=n_{I} \frac{V_{P}}{V_{Q}}\left(\frac{I_{s S}}{Q_{s s}}-1\right)
$$

In adults, studies indicate that the steady state interstitial to plasma insulin ratio $\left(Q_{s s} / I_{s s}\right)$ is between 0.4 and 0.6 [53-56]. In this study, a ratio of 0.5 is used.

If steady state conditions are also applied to Equation 2 then the sum of the kidney and liver clearances can be estimated: 


$$
n_{K}+n_{L} \approx \frac{-n_{I}\left(I_{s S}-Q_{s S}\right)+\frac{u_{e x}(t)}{V_{P} * m_{b o d y}}+\left(1-x_{L}\right) u_{e n}}{I_{s S}}
$$

\subsubsection{Kidney Clearance of Insulin}

It is estimated that $30-80 \%$ of insulin in the systemic circulation is removed by the kidneys [57]. Renal clearance of insulin occurs via two pathways: glomerular filtration, and absorption from the peritubular capillaries. Glomerular filtration compromises about $60 \%$ of total insulin clearance by the kidney [57]. Creatinine and inulin clearance are two common molecules used to estimate glomerular filtration rate (GFR). The rate of glomerular filtration of insulin is approximately $90 \%$ that of inulin determined GFR [58]. As such, total insulin clearance via the kidney can be estimated:

$$
n_{k}=\frac{1}{V_{P}} \times \frac{0.9 G F R_{\text {inulin }}}{0.6}
$$

Where $G F R_{\text {inulin }}$ is in units of $\mathrm{ml} / \mathrm{min}$, and $V_{P}$ is the distribution volume of insulin. For this cohort, GFR $R_{\text {inulin }}$ can be estimated using [59]:

$$
G F R_{\text {inulin }}=0.45+0.24 m_{b w}+0.18 A_{P N}
$$

Here $m_{b w}$ is the birth is weight, and $A_{P N}$ is the postnatal age in weeks.

\subsubsection{Insulin Volumes of Distribution}

Previously it was indirectly assumed that interstitial and blood plasma fluid volumes of distribution for insulin were essentially equal [37]. This assumption is not correct for neonates, and a literature search in PubMed was carried out to determine how plasma and interstitial fluid volumes change over time or between patients. As interstitial fluid volume is the extracellular fluid volume (ECV) minus the plasma volume $\left(V_{P}\right)$, extracellular fluid volumes were also included in the search. Key search terms included: plasma, extracellular, interstitial, fluid volume, and neonate. Exclusion criteria included lack of reported values, non-human or adult fluid volumes reported. Fluid volumes were analysed in units of $\mathrm{mL} / \mathrm{kg}$. Results were plotted against postnatal age and reported standard deviations were included as error bars. 


\subsection{Clinical Data and Model Validation}

\subsubsection{C-peptide Data}

The clinical data used here has been described in detail elsewhere $[49,50]$. In summary, three to four blood samples were taken from 88 hyperglycaemic (two BG measures $>8.5 \mathrm{mmol} / \mathrm{L}$ more than 4 hours apart) very preterm (born at $<32$ weeks gestation) and/or very low birth weight $(<1500 \mathrm{~g})$ infants enrolled in a randomised trial of glycaemic control (the HINT trial [28], Auckland, New Zealand). Blood samples were taken at randomisation (0-5 days post natal age) and at approximately 7, 14 and 21 days after randomisation.

Plasma insulin (Azsym system auto-analyzer, Abbott Laboratories, Abbott Park, IL) and glucose (glucose oxidise method, ABL 700, Radiometer Ltd, Copenhagen, Denmark) concentrations were determined as part of the study, and remaining blood from the samples was frozen. Of the original cohort of 88,41 were of GA<32 weeks and had 1 or more samples with sufficient remaining blood volume available for retrospective C-peptide analysis. C-peptide concentrations were determined using immunometric assays (Elecsys 2010, Roche Diagnostics, Germany). Table 2 provides demographic data over the sample cohort, as well raw sample results.

\subsubsection{Model Validation Cohorts}

The model was validated through retrospective fitting to clinically charted glycaemic data from two New Zealand NICU cohorts. The Christchurch Women's Hospital (CWH) cohort consists of 67 very/extremely premature patients who received insulin either under sliding scale-based or modelbased protocols [60]. The HINT cohort [28] was derived from detailed BG, insulin and nutrition data charted during the HINT trial in Auckland, NZ. This is the same cohort from which the C-peptide data was obtained, but those one-off weekly blood samples did not always appear in the clinical BG record. Of a total of 88 patients in the trial, 55 had sufficiently detailed data (BG measured at least 6 hourly, generally more frequently) to extract one or more glycaemic episodes per subject. A glycaemic episode is defined as $\geq 12$ hours of insulin therapy, and at least 4 BG (max 6 hours apart) 
measurements during this time. These criteria resulted in a total of 243 patient episodes across both cohorts, with all details in Table 3.

$S_{I}$ profiles were fit to clinical data from the CWH and HINT validation cohorts using the integral based fitting method [60] and Equations 1-4. Ordinary differential equation solutions for Equations 1 $3(G, I, Q)$ were generated using a Runga-Kutta 4 based method (Matlab ${ }^{\circledR}$, ode45). Insulin kinetic parameter values determined in this analysis $\left(n_{L}, n_{k}, n_{I}, n_{c}, V_{P}, V_{Q}\right)$ were used to generate the plasma and interstitial insulin concentrations $(I, Q)$. The identified $S_{I}$ must remain positive to be physiologically correct, so the lower limit of $S_{I}$ was set to $1 \times 10^{-7} \mathrm{~L} / \mathrm{mU} / \mathrm{min}$ (near 0 , where a typical $S_{I}$ is $10^{-4}-10^{-3} \mathrm{~L} / \mathrm{mU} / \mathrm{min}$ ). For comparison, $S_{I}$ profiles were also fit, and BG and insulin profiles generated, using the original NICU model [37]. Fitting and prediction error were compared between the older NICU model iteration and the new NICING model as a measure of validation to show similar performance to a working, clinically effective standard.

Table 2: Clinical and sample characteristics. Numbers are presented as median [IQR] or number (\% of total).

\begin{tabular}{lc}
\hline Number of Patients & \\
Total & 41 \\
Control & 21 \\
Tight Glycaemic Control & 20 \\
Male (\%) & $20(49 \%)$ \\
Age $\quad$ Gestational, weeks & $27.2[26.2-28.7]$ \\
$\quad$ Post natal, days & $9.5[4-17]$ \\
Birth weight & \\
grams & $839[735-1000]$ \\
Z score & $-0.19[-1.03-0.14]$ \\
Small for gestational age & 6 \\
Ethnicity & \\
Asian & $9(22 \%)$ \\
Caucasian & $11(27 \%)$ \\
Maori & $17(41 \%)$ \\
Pacific Island & $4(10 \%)$ \\
Sample results & \\
Number of Samples & 54 \\
Day after randomisation* & $7[0-14]$ \\
BG, mmol/l & $7.5[5.1-10.5]$ \\
Plasma insulin concentration, pmol/L & $59.0[99.3-181.9]$ \\
Plasma C-peptide concentration, $\mathrm{nmol} / \mathrm{L}$ & $2.3[1.1-4.2]$ \\
\hline
\end{tabular}

*Samples were taken on day of randomisation 0,7 , and 14 . 
Table 3: Patient cohort characteristics, presented as number of patients, or median [interquartile range].

\begin{tabular}{lcc}
\hline & HINT & CWH \\
\hline Patients & 55 & 67 \\
Total Number & $33 / 22$ & $22 / 45$ \\
Control/TGC & $27 / 28$ & \\
Male/female & & \\
Age & $25[24-26]$ & $27[25-28]$ \\
Gestational age, weeks & $4[3-8]$ & $2[0-5]$ \\
Post natal age, days & & \\
Birth weight & $690[740-890]$ & $865[690-960]$ \\
grams & $0.02[-0.85-0.58]$ & $-0.67[-1.2-0.27]$ \\
Z score & 9 & 13 \\
SGA & 160 & 83 \\
Total Episodes & $57 / 103$ & $25 / 48$ \\
Control/TGC & 4673 & 8455 \\
Total hours & 1389 & 2951 \\
Total BG measurements & $4.0[3.5-4.8]$ & $3.3[2.8-3.9]$ \\
Median time between measurements & $7.10[4.90-9.40]$ & $7.10[5.70-9.00]$ \\
(hours) [IQR] & $0.060[0.033-0.080]$ & $0.036[0.026-0.051]$ \\
Median BG [mmol/L] & & \\
Median insulin rate [IQR] (U/kg/hr): &
\end{tabular}

\subsubsection{Fitting and Prediction Error}

Fitting error was calculated as the percentage difference between the model solution at a measurement time and the actual measured value. Where fitting error is high, the model fails to fit clinical data and is therefore inappropriate. However, for appropriately constructed model dynamics, fitting error saturates at some lower value as the fitted insulin sensitivity ensures model fit over a reasonable range of parameter values [60]. For this reason, prediction error is also analysed.

Prediction error is calculated as the percentage difference between the actual measured BG and a 1 hour forward prediction, where 1 hour is the minimum resolution of $S_{I}$ in this framework. The prediction from time $t_{n}$ to $t_{n+1}$ is based on $S_{I}$ from $t_{n-1}$ to $t_{n}$ and the $50^{\text {th }}$ percentile (median likely outcome) of observed outcomes based on statistical models of a base population [61]. Lower prediction error indicates that dynamics previously reflected in $S_{I}$ are now more comprehensively modelled. As a result, there is improved ability of the model to forward predict median likely changes in BG for a given insulin-nutrition treatment, which is essential for accurately assessing future patientspecific state, and thus for effective control. 


\subsection{Results}

\subsection{Fluid Volume Analysis}

A total of 10 studies $(n=18-32$, exception [62] with $n=5)$ examined typical fluid volumes in preterm neonates with an average study cohort gestation age $<33$ weeks and birth weight $<2 \mathrm{~kg}$. Of these, 7 reported ECV values [62-67] and 4 reported $V_{P}[62,68-70]$. Figure 1 shows a significant drop in ECV over the first 1-2 weeks post natal age (PNA), followed by a relative plateau. Although a lot of variability is seen between study mean values, most studies fall within one standard deviation of each other.

Given this data, a power law function $\left(\mathrm{R}^{2}=0.44\right)$ was fit to capture the rapid decrease in ECV over the first few days and subsequent ECV plateau:

$$
E C V=492 \times P N A^{-0.09}
$$

Figure 2 shows that plasma volume does not significantly change over the first couple of weeks of life. While Ussher and Lind [70] show a very slight decrease over time $\left(\mathrm{R}^{2}=0.04\right)$, Bauer et al [62] shows a slight increase. Therefore, $V_{P}$ could be assumed to remain essentially constant over time, with an average volume of $47 \mathrm{~mL} / \mathrm{kg}$. The interstitial fluid volume is thus:

$$
V_{Q}=492 \times P N A^{-0.09}-V_{P}
$$




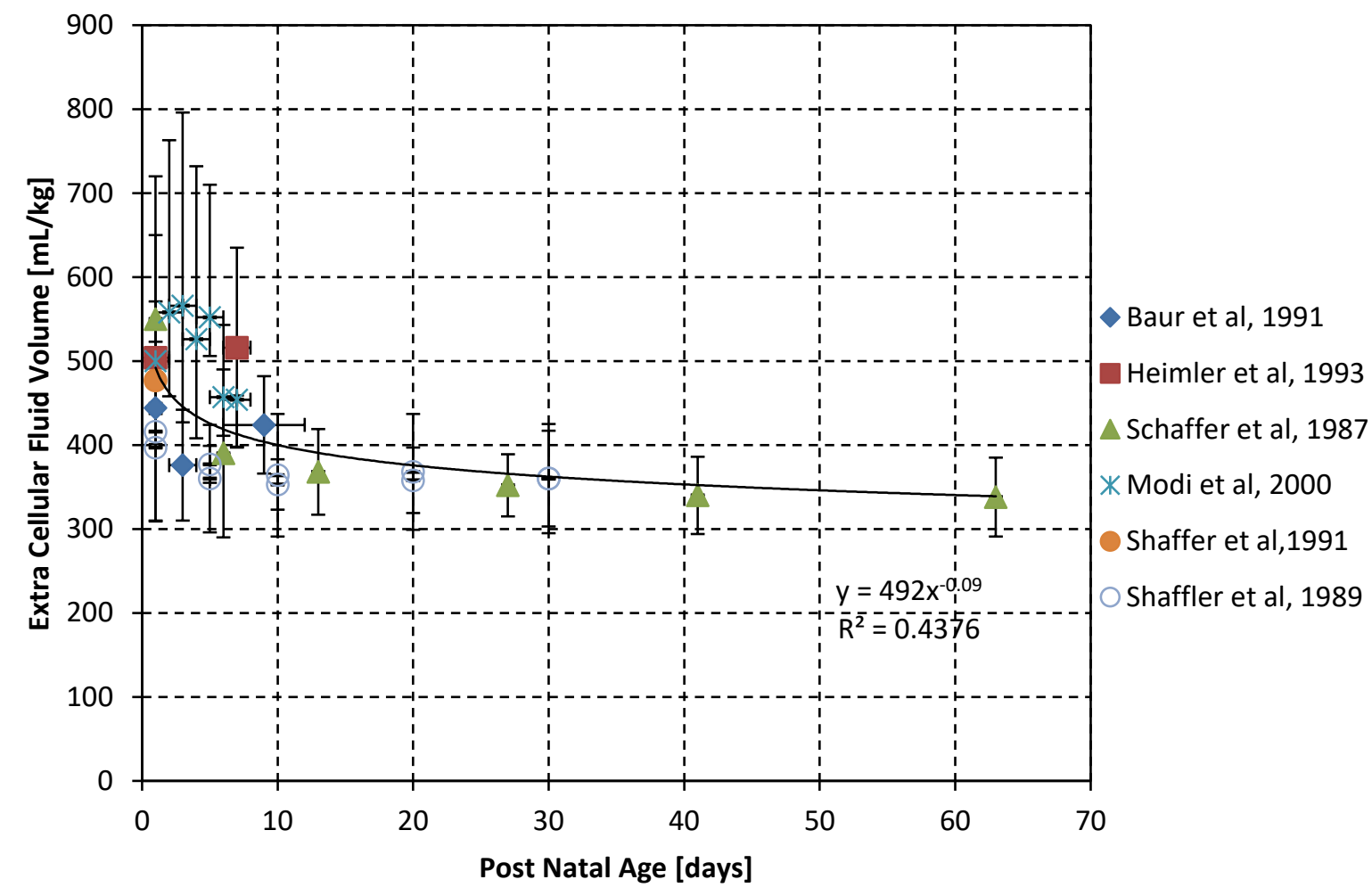

Figure 1: Decrease of ECV with postnatal age. Vertical Error bars are 1 standard deviation; horizontal error bars cover the range of days. For the Modi et al data, the median and range is plotted.

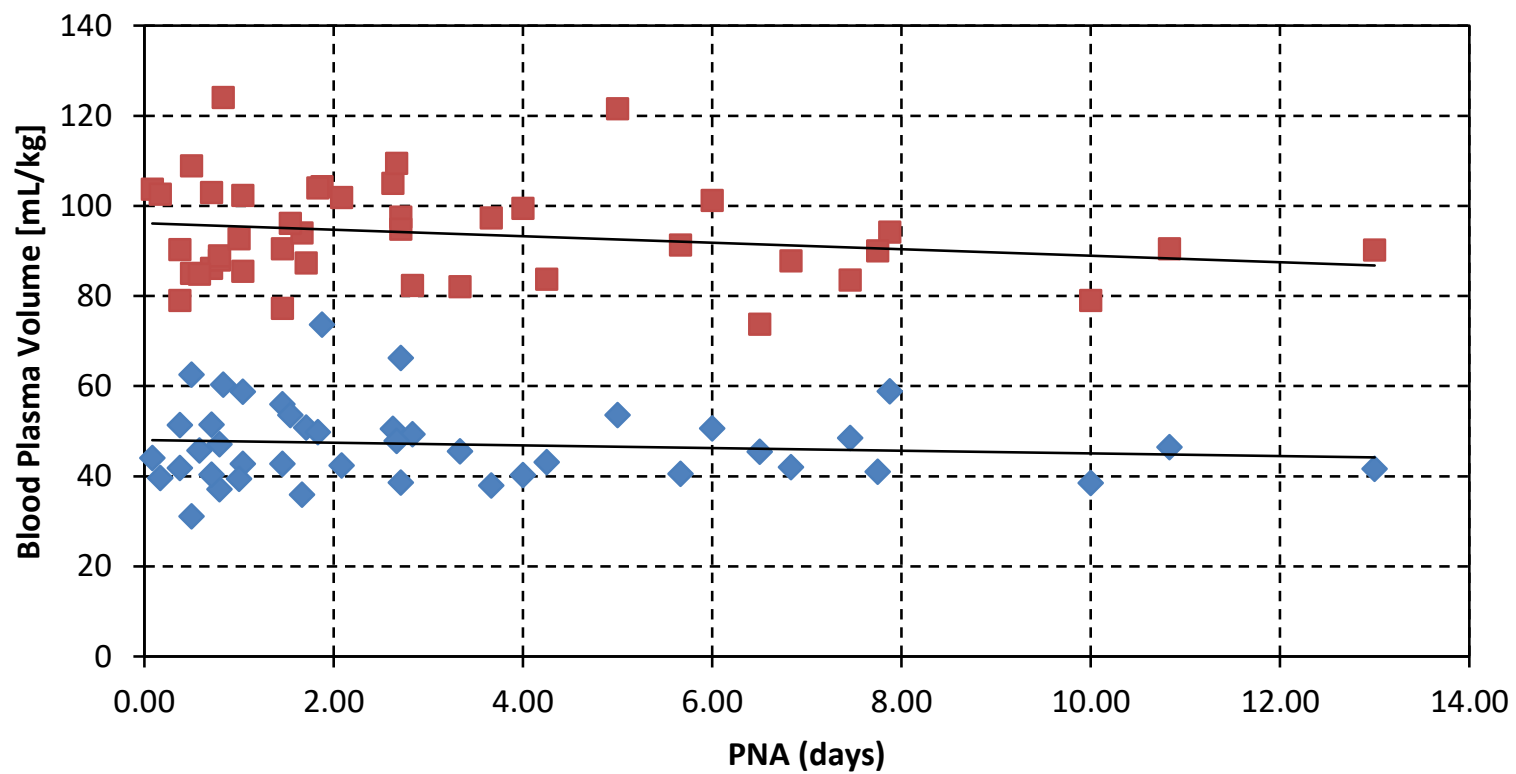

Figure 2: Plasma (४) and blood volume ( $\square$ ) changes over time. Data used is patients with BW $<2$ KG from Ussher et al [70]. 


\subsection{Inference of Insulin Clearance Parameters}

The results from the evaluation of Equations 14-18, using C-peptide data to estimate insulin secretion, are presented in Table 4 and Figure 3. It should be noted that $n_{C}$ is scaled by the ratio of plasma volume to interstitial volume, as per Equation 17. The parameter $n_{K}$ is made patient specific and dependant on a birth weight and postnatal age dependant model of GFR, according to Equations 1920. The distribution of patient specific values for liver clearance, $n_{L}$, calculated using Equation 18 , is shown in Figure 3, with a median clearance of $0.39 \mathrm{~min}^{-1}$.

Sensitivity to assumptions around diffusion between compartments and hepatic insulin clearance was tested and results are shown in Figure 4. As $n_{I}$ is an order of magnitude smaller than $n_{L}$, changes in $n_{I}$ had very little effect on patient specific results for $n_{L}$. Figure 4 shows that within the sensitivity range, $n_{I}$ had little effect on modelled plasma insulin concentrations. Model fit error was not affected, as the small change in peripheral insulin concentration, $Q$, resulted in a scale offset in $S_{I}$. The hepatic insulin clearance parameter, $n_{L}$, is much more sensitive, as evidenced in Figure $4 \mathrm{~b}$ ). This aligns with expectations from Figure 3, where liver clearance was observed to be highly patient specific.

Table 4: Clearance Parameters based on HINT C-peptide analysis and physiological ranges in adult subjects. Unless otherwise state, results are presented as Median [IQR]

\begin{tabular}{|c|c|c|}
\hline Parameter & Value [1/min] & $\begin{array}{c}\text { Physiological range } \\
\text { for adults [Lotz] } \\
\text { Median (Range) [71] }\end{array}$ \\
\hline$n_{I}$ & 0.025 & $\begin{array}{c}0.28[0.22-0.36][\mathbf{L} / \mathbf{m i n}] \\
0.0052[0.0041-0.0119] \\
{[\mathbf{1} / \mathbf{m i n}]^{*}}\end{array}$ \\
\hline$n_{K}$ & $\begin{array}{c}0.034 \\
{[0.030-0.039]}\end{array}$ & $0.06(0.053-0.064)$ \\
\hline$n_{L}$ & $\begin{array}{c}0.39 \\
{[0.15-0.70]}\end{array}$ & $0.15(0.1-0.21)$ \\
\hline$n_{c} \frac{V_{Q}}{V_{P}}=n_{I}\left(\frac{I_{S S}}{Q_{s S}}-1\right)$ & 0.025 & - \\
\hline
\end{tabular}

* From [72] 


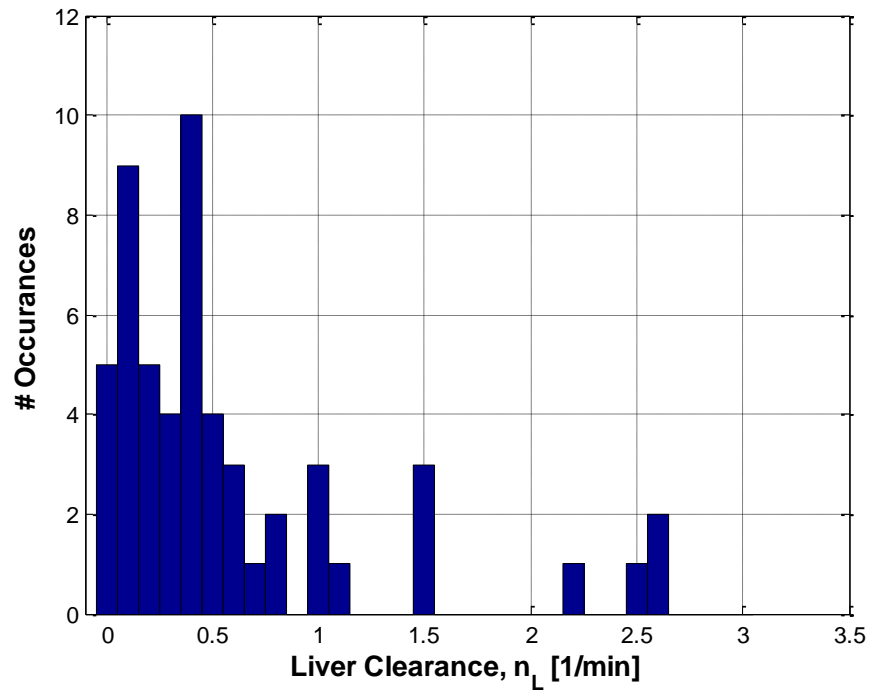

Figure 3: Liver clearance parameter, $n_{L}$, distribution derived from HINT data.
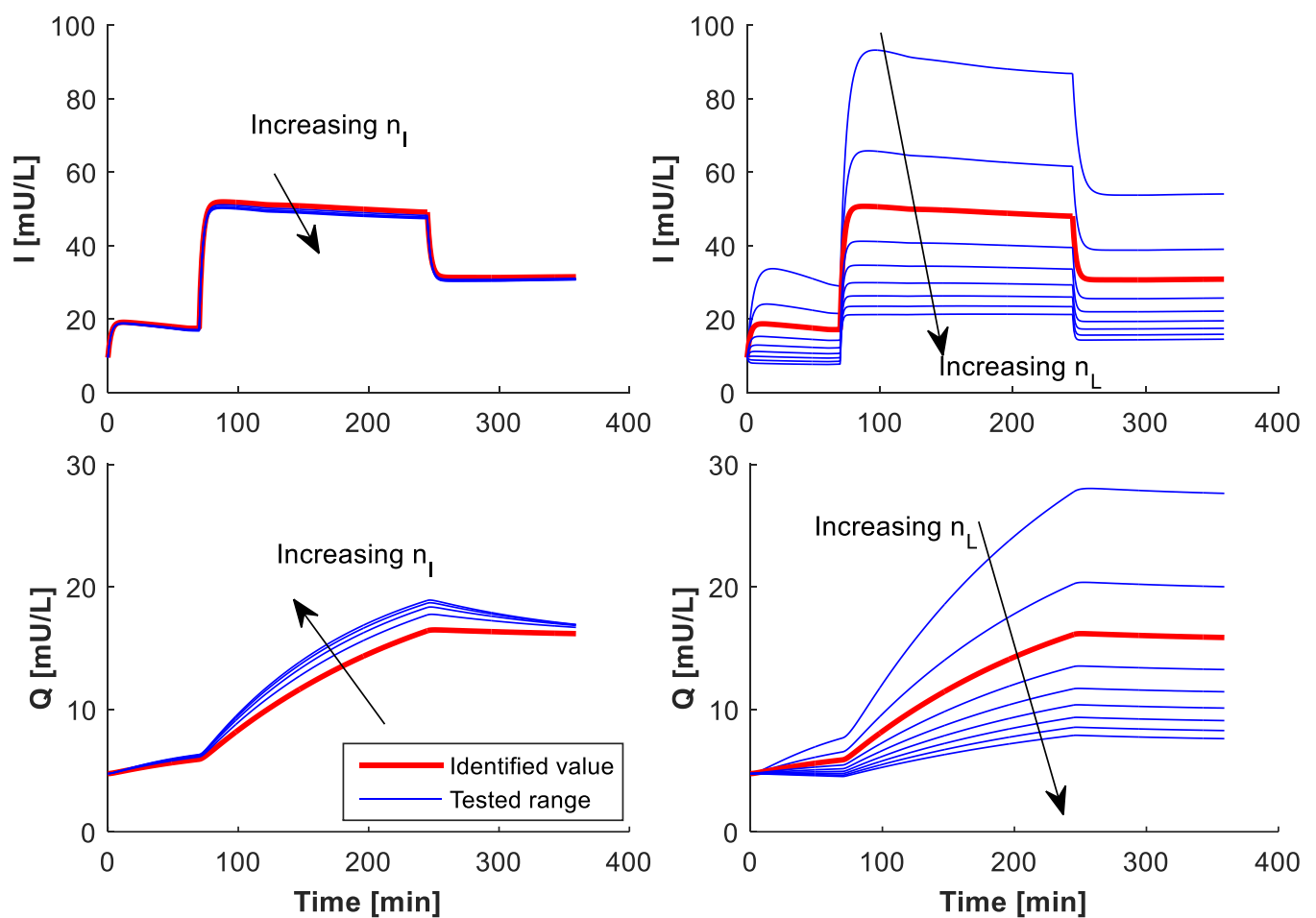

a)

b)

Figure 4: Sensitivity analysis of on a) diffusion of insulin between compartments $\left(n_{I}\right)$, and b) hepatic insulin clearance $n_{L}$. Increasing $n_{I}$ was achieved by multiplying the mass ratio of Equation 12 by a power of $[1 / 1,1 / 1.5,1 / 2,1 / 2.5,1 / 3]$, giving $n_{I}$ in the range $0.025-0.038$ 1/min. $n_{L}$ was varied across the range: $[0.20: 0.10: 1] 1 / \mathrm{min}$. 
Table 5 presents the fitting and prediction error results over the HINT and CWH patient cohorts for both the new NICING and the previous NICU [45] models. There was no difference in fitting error between the two models in the CWH cohort, and prediction error was slightly increased. In the HINT cohort, both fitting and prediction error were slightly decreased with the NICING model. All changes were very small relative to typical measurement errors. Figure 5 shows two examples fits to clinical data. Model fit is good, with only a very small undershoot on some peaks.

Table 5: Fitting and Prediction error for the NICING and NICU models

\begin{tabular}{|l|l|l|}
\hline & CWH Cohort & HINT Cohort \\
\hline NICING model & & \\
\hline Fitting Error & $2.1 \%$ & $2.7 \%$ \\
\hline Prediction Error & $12.6[5.2-24.7] \%$ & $18.3[7.3-34.0] \%$ \\
\hline NICU model & & \\
\hline Fitting Error & $2.1 \%$ & $2.9 \%$ \\
\hline Prediction Error & $11.7[5.1-23.5] \%$ & $18.8[8.1-37.0] \%$ \\
\hline & & \\
\hline
\end{tabular}
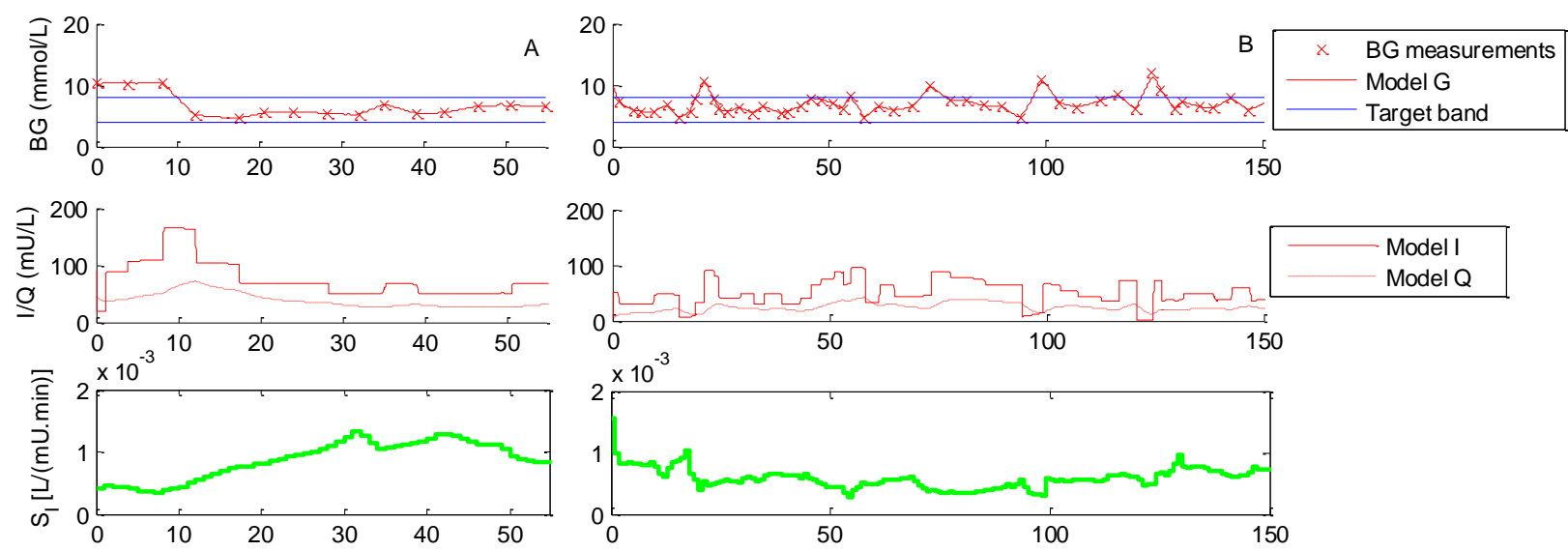

Time [hours]

Figure 5: Model fit in A: a very stable shorter-stay patient, and B: long-stay dynamic patient. 


\subsection{Discussion}

This study presents a more physiologically descriptive glucose-insulin model for the purposes of glycaemic control in premature infants. In particular, it focuses on the kinetics of insulin, which are necessary to obtain accurate estimates of patient-specific metabolic state, and thus accurate control. Previously in the NICU model [45], insulin clearance via the liver, kidney, and receptor-bound degradation was modelled by a single clearance parameter determined from literature data and minimisation of model fitting error. The NICING model separates out these clearances, adding a degradation term to the interstitial compartment, saturable liver clearance, and kidney clearance [38]. Birth weight and age based modelling of plasma volumes and GFR allow inter-compartment transport and renal clearances to be-patient appropriate.

After these changes, the full NICING model system has similar or better ability to fit two cohorts of clinical data when compared to a simpler and less physiologically relevant previous iteration of the model, as evidenced by the unchanged fitting error in the CWH cohort and the reduction in the HINT cohort. This reflects the model's ability to capture time-varying dynamics in the wider HINT cohort from which the insulin clearance parameters were derived, as well as in a separate, independent cohort to test whether the new model parameters were effective in capturing observed clinical dynamics. Prediction error was increased by $\sim 1 \%$ in the $\mathrm{CWH}$ cohort, and decreased by $\sim 1 \%$ in the HINT cohort, suggesting that the NICING model overall is likely to perform in a very similar manner to the NICU model. Clinical results to date using the old NICU model in model-based control [73, 74] suggest that it is robust, safe, and effective for use in glycaemic control in a NICU setting [74-76].

The NICING model is used in a risk-based decision framework $[74,77]$. Hourly identification of $S_{I}$ allows changes in insulin sensitivity to be observed. Stochastic modelling is used to predict future changes in insulin sensitivity, resulting in a distribution of likely BG outcomes for a given intervention. Insulin interventions are chosen such that this range of likely BG outcomes overlaps with the desired clinical targets. Specifically, we set a maximum risk threshold for mild hypoglycaemia $(\mathrm{BG}<4.0 \mathrm{mmol} / \mathrm{L})$ at $5 \%$. New results to date using the NICING model show further 
significant improvement in control and safety [73] over the previous NICU model, indicating that the new model more accurately captures underlying dynamics, beyond what can be evaluated using fitting and prediction error.

Most of the insulin clearance parameters lie within the reported physiological range for adults, suggesting these volume normalised values are reasonable. Insulin diffusion between the plasma and peripheral compartments, $n_{I}$, is an order of magnitude faster than the adult ICING model $\left(n_{I}=\right.$ $0.003 \mathrm{~min}^{-1}$ ). The associated half life of action for $n_{I}$ is $\sim 150$ minutes, and when $n_{C}$ is also considered, the combined time to half maximal interstitial insulin for an IV insulin infusion is $\sim 70$ minutes, which is similar to results from studies in lean and obese adults (46 and 72 minutes respectively) [56]. Given that that the ICING model value for $n_{I}$ was chosen based on extensive grid search and fitting error minimisation [5], it is likely that the NICING value is more physiologically real.

Insulin kinetic parameters were assumed to be independent of nutritional intake. Previous work saw no clear trend or effect of nutrition on insulin secretion [49,50], which is known in adults to be more directly affected by nutritional intake and resulting hormone signalling.

Hepatic liver clearance was found to be highly patient specific (Figure 3), and 2-3 times higher than adult values [71]. The wide range of clearance values found are a reflection of the model's sensitivity to this parameter (Figure 4), where, having calculated all other parameters, $n_{L}$ was fit such that the model fit the samples plasma insulin concentration. This variability could also result from accumulated patient-specific deviation from other model parameters, however given kidney clearance $\left(n_{K}\right)$ and diffusion between compartments $\left(n_{I}\right)$ are an order of magnitude smaller, this effect is not likely to be significant. The high variability in the results is similar to the large variability in endogenous glucose production seen in previous work [39], and likely a reflection of patient condition and metabolic maturity in this very premature cohort. Patient-specific deviations in hepatic insulin clearances may thus result in a bias in the overall $S_{I}$ profile for some patients, but are not likely to affect short-term changes in $S_{I}$. 
The higher liver clearance constant in this premature infant cohort could be a reflection of overall higher hepatic metabolism and/or the relatively larger proportional mass of the infant liver. However, this is speculative as no studies specifically examine hepatic insulin kinetics in the premature infant. Premature infants have larger proportional organ mass and higher basal metabolic rate in proportion to total body mass than adults [78]. Previous reviews of literature show that endogenous glucose production (EGP) is generally higher in premature infants (median $2.2 \mathrm{mg} / \mathrm{kg} / \mathrm{min}$ [39] vs. mean 1.4 $\mathrm{mg} / \mathrm{kg} / \mathrm{min}$ in unfasted adults [79], EGP is higher at $\sim 4 \mathrm{mg} / \mathrm{kg} / \mathrm{min}$ in fasted adults [79]) and is also unsuppressed at higher $\mathrm{BG}$ and/or glucose infusion rate in premature infants [80]. Infants born less than 27 weeks gestation lack glycogen stores, so intravenous/frequent exogenous nutrition is required to meet energy needs. Thus, the premature liver may utilise and eventually clear more insulin than its adult counterpart, and for this reason liver clearance of insulin may be higher.

Birth weight and age based modelling of plasma volumes and GFR allow inter-compartment transport and renal clearances to be patient-appropriate. This approach is in contrast to the adult case, where these volumes are held constant. In addition, during the first week of life there is significant weight loss in the premature infant as the extracellular fluid compartment contracts. Modelling this contraction will prevent long term bias in identified $S_{I}$ profiles, thus providing more accurate $S_{I}$ estimates for use in glycaemic control.

Insulin degradation is being modelled as a process that is unsaturated at high insulin concentrations. [42] observed insulin mediated glucose uptake was not saturated with insulin concentration. Insulin mediated glucose uptake is a receptor-mediated process, and receptor-bound insulin is either released back into the extracellular space, or internalised by the cell [43]. As such, the same saturation parameter is applied to both insulin-mediated glucose uptake and insulin degradation. In this case it has been set to zero, due to the lack of observed saturation of insulin-mediated glucose uptake.

Glycaemic control using the ICING model in the adult intensive care unit has proven safe and effective, with low incidence of hypoglycaemia and around $90 \%$ time in a clinically targeted range of 4.0-8.0mmol/L [3]. The NICING model is based on the ICING model, and should thus allow 
consistency with existing adult ICU glycaemic control protocols and T1DM/T2DM orientated models [81]. The greater physiological descriptiveness of the NICING model in comparison to the old NICU model additionally allows it to be more easily customised to the infant case using clinical C-peptide data, when available, and future metabolic studies.

Study limitations include the assumption of steady state kinetics in the analysis of C-peptide concentrations. Due to the inherent extreme fragility of this premature neonatal cohort, serial blood samples are not practically or ethically possible, so the more detailed model based analysis of Cpeptide and insulin kinetics seen in adult studies [48, 82] are not possible. Such steady-state assumptions are not unreasonable in this cohort, as the majority of nutrition is given via parenteral infusion, or enteral feeds, which do not tend to change dramatically from hour to hour. In addition, none of the samples were taken within 2 hours of an infant starting insulin infusion. Hence, the assumption of steady state conditions is both reasonable and a necessity in this cohort.

Further, no kinetic parameters are available for C-peptide dynamics specific to this cohort, so adult kinetic parameters, adjusted for age, were used. Patient specific parameters for neonates cannot be determined due to the demands of the procedure. While it is understandably not desirable to use age to extrapolate these adult parameters this far beyond the cohort for which they were originally validated, the resulting normalised parameter values fall within ranges reported in studies on adults [48, 51]. This outcome provides a level of qualitative validation that the assumption used is not poor. In addition, using adult kinetic population constants, particularly under the assumption of steady state, is not likely to change any of the underlying trends observed, and scale differences are absorbed by the model in an overall shift in $S_{I}$ without altering control outputs. Thus, as long as $S_{I}$ forecasting models are scaled consistently, relative changes in $S_{I}$, enabling BG prediction, are accounted for. If this model were to be used in a more detail-oriented setting (e.g. like the metabolic analysis of $[1,2]$ ), or if patient-specific insulin parameters (e.g. liver clearance), were to be identified in real time for control, this assumption may require revisiting and further examination. This study highlights the need for data and studies to validate any significant differences in neonatal kinetics that would affect this assumption, as well as, more generally, the potential value of pursuing such studies in this cohort. 
A limitation of the HINT data used in validation is that all nutritional data was recorded as daily totals. It was assumed that daily totals were delivered as a constant infusion over the course of the day, which is not unreasonable in this cohort. However, the slightly higher fitting error in this cohort is likely caused in part by this assumption.

\subsection{Conclusions}

A physiological model of glucose-insulin kinetics for use in glycaemic control in very premature infants is presented. The insulin kinetics model is more physiologically descriptive than previously, and parameters identified using a novel set of C-peptide concentrations from very premature infants. Cohort and infant-specific insulin clearance parameters were identified for clearance through the liver, the kidneys, and peripheral degradation. Saturation of liver clearance at high insulin concentration is modelled, and kidney clearance is modelled according to changes in kidney function with birth weight and gestational age. Estimations of insulin distribution values based on fluid compartments are found from literature data, and the post-natal contraction of the extracellular fluid compartment is also captured. Fitting error between the model and clinical validation data across two clinical data sets indicate that this model has a similar or better ability to fit clinical data compared to a previous, less physiologically detailed and relevant NICU model, and tight glycaemic control performance is significantly improved.

\subsection{Acknowledgements and conflicts of interest}

The authors declare that there are no conflicts of interest associated with this work. Clinical data from the HINT trial was provided by Dr Jane Alsweiler, and analysed independently from the original trial. Additional C-peptide analyses on existing blood samples was funded by Health Research Council of New Zealand. Clinical data from Christchurch Women's Hospital was collected as part of ongoing research between the University of Canterbury and Christchurch Women's Hospital. The main body of this work and manuscript was carried out by Jennifer Dickson, who was funded by a Canterbury $\mathrm{PhD}$ Scholarship. 


\subsection{References}

1. Hovorka, R., et al., Nonlinear model predictive control of glucose concentration in subjects with type 1 diabetes. Physiological Measurement, 2004. 25(4): p. 905-920.

2. Carson, E.R. and C. Cobelli, Modelling methodology for physiology and medicine. Academic Press Series in Biomedical Engineering2001, San Diego: Academic Press. xiv, 421.

3. Fisk, L., et al., STAR Development and Protocol Comparison. IEEE Trans Biomed Eng, 2012. 59(12): p. 3357-3364.

4. Van Herpe, T., et al., A minimal model for glycemia control in critically ill patients. Conf Proc IEEE Eng Med Biol Soc, 2006. 1: p. 5432-5.

5. Lin, J., et al., A physiological Intensive Control Insulin-Nutrition-Glucose (ICING) model validated in critically ill patients. Comput Methods Programs Biomed, 2011. 102(2): p. 192205.

6. Terranova, N., et al., Assessing the Glucose Time Course in Critically III Patients by a Mathematical Model, in 5th European Conference of the International Federation for Medical and Biological Engineering: 14-18 September 2011, Budapest, Hungary, Á. Jobbágy, Editor 2012, Springer Berlin Heidelberg: Berlin, Heidelberg. p. 299-302.

7. Man, C.D., et al., The UVA/PADOVA Type 1 Diabetes Simulator: New Features. Journal of diabetes science and technology, 2014. 8(1): p. 26-34.

8. Docherty, P.D., et al., A graphical method for practical and informative identifiability analyses of physiological models: a case study of insulin kinetics and sensitivity. Biomed Eng Online, 2011. 10: p. 39.

9. Chase, J.G., et al., Model-based glycaemic control in critical care - a review of the state of the possible. Biomedical Signal Processing and Control, 2006. 1(1): p. 3-21.

10. Chase, J.G., et al., Tight glycemic control in critical care - The leading role of insulin sensitivity and patient variability: A review and model-based analysis. Computer Methods and Programs in Biomedicine, 2011. 102(2): p. 156-171.

11. Alsweiler, J.M., C.A. Kuschel, and F.H. Bloomfield, Survey of the management of neonatal hyperglycaemia in Australasia. Journal of Paediatrics and Child Health, 2007. 43(9): p. 632635.

12. Dweck, H.S. and G. Cassady, Glucose Intolerance in Infants of Very Low Birth Weight. Pediatrics, 1974. 53(2): p. 189.

13. Louik, C., et al., Risk factors for neonatal hyperglycemia associated with $10 \%$ dextrose infusion. American Journal of Diseases of Children, 1985. 139(8): p. 783-6.

14. Heimann, K., et al., Are recurrent hyperglycemic episodes and median blood glucose level a prognostic factor for increased morbidity and mortality in premature infants $</=1500 \mathrm{~g}$ ? Journal of perinatal medicine, 2007. 35(3): p. 245-8.

15. Hays, S.P., B. Smith, and A.L. Sunehag, Hyperglycemia Is a Risk Factor for Early Death and Morbidity in Extremely Low Birth-Weight Infants. Pediatrics, 2006. 118(5): p. 1811-1818.

16. Hall, N.J., et al., Hyperglycemia is associated with increased morbidity and mortality rates in neonates with necrotizing enterocolitis. Journal of pediatric surgery, 2004. 39(6): p. 898-901; discussion 898-901.

17. Kao, L.S., et al., Hyperglycemia and morbidity and mortality in extremely low birth weight infants. Journal of perinatology, 2006. 26(12): p. 730-6.

18. Alaedeen, D.I., M.C. Walsh, and W.J. Chwals, Total parenteral nutrition-associated hyperglycemia correlates with prolonged mechanical ventilation and hospital stay in septic infants. J Pediatr Surg, 2006. 41(1): p. 239-44.

19. Bistrian, B.R., Hyperglycemia and infection: which is the chicken and which is the egg? JPEN J Parenter Enteral Nutr, 2001. 25(4): p. 180-181.

20. Blanco, C.L., et al., Hyperglycemia in extremely low birth weight infants in a predominantly Hispanic population and related morbidities. Journal of Perinatology, 2006. 26(12): p. 737741. 
21. Garg, R., et al., Hyperglycemia and retinopathy of prematurity in very low birth weight infants. Journal of perinatology, 2003. 23(3): p. 186-94.

22. Binder, N.D., et al., Insulin infusion with parenteral nutrition in extremely low birth weight infants with hyperglycemia. The Journal of pediatrics, 1989. 114(2): p. 273-80.

23. Collins, J.W., Jr., et al., A controlled trial of insulin infusion and parenteral nutrition in extremely low birth weight infants with glucose intolerance. The Journal of pediatrics, 1991. 118(6): p. 921-7.

24. Meetze, W., et al., Hyperglycemia in extremely-low-birth-weight infants. Biol Neonate, 1998. 74(3): p. 214-21.

25. Ostertag, S., et al., Insulin pump therapy in the very low birth weight infant. Pediatrics, 1986. 78(4): p. 625-630.

26. Pollak, A., et al., Glucose Disposal in Low-Birth-Weight Infants During Steady State Hyperglycemia: Effects of Exogenous Insulin Administration. Pediatrics, 1978. 61(4): p. 546.

27. Vaucher, Y.E., P.D. Walson, and G. Morrow, 3rd, Continuous insulin infusion in hyperglycemic, very low birth weight infants. Journal of pediatric gastroenterology and nutrition, 1982. 1(2): p. 211-7.

28. Alsweiler, J.M., J.E. Harding, and F.H. Bloomfield, Tight glycemic control with insulin in hyperglycemic preterm babies: a randomized controlled trial. Pediatrics, 2012. 129(4): p. 639-47.

29. Ng, S.M., J.E. May, and A.J. Emmerson, Continuous insulin infusion in hyperglycaemic extremely-low- birth-weight neonates. Biol Neonate, 2005. 87(4): p. 269-72.

30. Beardsall, K., et al., Early elective insulin therapy can reduce hyperglycemia and increase insulin-like growth factor-l levels in very low birth weight infants. The Journal of pediatrics, 2007. 151(6): p. 611-7.

31. Bagshaw, S., et al., The impact of early hypoglycemia and blood glucose variability on outcome in critical illness. Critical Care, 2009. 13(3): p. R91.

32. Krinsley, J.S., et al., Mild hypoglycemia is independently associated with increased mortality in the critically ill. Critical care, 2011. 15(4): p. R173.

33. Egi, M., et al., Hypoglycemia and outcome in critically ill patients. Mayo Clin Proc, 2010. 85(3): p. 217-24.

34. Lucas, A., R. Morley, and T.J. Cole, Adverse neurodevelopmental outcome of moderate neonatal hypoglycaemia. British Medical Journal, 1988. 297(6659): p. 1304-1308.

35. Koh, T.H., et al., Neural dysfunction during hypoglycaemia. Arch Dis Child, 1988. 63(11): p. 1353-8.

36. Farrag, H.M. and R.M. Cowett, Glucose homeostasis in the micropremie. Clinics in Perinatology, 2000. 27(1): p. 1-22.

37. Le Compte, A.J., et al., Modeling the glucose regulatory system in extreme preterm infants. Comput Methods Programs Biomed, 2011. 102(3): p. 253-66.

38. Dickson, J.L., et al., Modelling Insulin Clearance and Degradation in Very Premature Infants, in 9th IFAC Symposium on Biological and Medical Systems.2015, IFAC Papers Online: Berlin.

39. Dickson, J.L., et al., On the problem of patient-specific endogenous glucose production in neonates on stochastic targeted glycemic control. J Diabetes Sci Technol, 2013. 7(4): p. 91327.

40. Gunn, C.A., et al., Brain mass estimation by head circumference and body mass methods in neonatal glycaemic modelling and control. Computer methods and programs in biomedicine, 2014. 115(2): p. 47-54.

41. Pielmeier, U., et al., A simulation model of insulin saturation and glucose balance for glycemic control in ICU patients. Computer Methods and Programs in Biomedicine, 2010. 97(3): p. 211-222.

42. Farrag, H.M., et al., Persistent glucose production and greater peripheral sensitivity to insulin in the neonate vs. the adult. Am J Physiol, 1997. 272(1 Pt 1): p. E86-93. 
43. Duckworth, W.C., R.G. Bennett, and F.G. Hamel, Insulin degradation: progress and potential. Endocr Rev, 1998. 19(5): p. 608-24.

44. Duckworth, W.C., F.G. Hamel, and D.E. Peavy, Hepatic metabolism of insulin. Am J Med, 1988. 85(5A): p. 71-6.

45. Le Compte, A., et al., Modeling the glucose regulatory system in extreme preterm infants. Computer methods and programs in biomedicine, 2011. 102(3): p. 253-66.

46. Hamilton-Wessler, M., et al., Mode of transcapillary transport of insulin and insulin analog NN304 in dog hindlimb: evidence for passive diffusion. Diabetes, 2002. 51(3): p. 574-82.

47. Steil, G.M., et al., Transendothelial insulin transport is not saturable in vivo. No evidence for a receptor-mediated process. J Clin Invest, 1996. 97(6): p. 1497-503.

48. Van Cauter, E., et al., Estimation of insulin secretion rates from C-peptide levels. Comparison of individual and standard kinetic parameters for C-peptide clearance. Diabetes, 1992. 41(3): p. 368-77.

49. Dickson, J.L., et al., A C-Peptide-Based Model of Pancreatic Insulin Secretion in Extremely Preterm Neonates in Intensive Care. Journal of diabetes science and technology, 2015.

50. Dickson, J.L., et al., Hyperglycaemic preterm babies have sex differences in insulin secretion. Neonatology, 2015. (in press).

51. Polonsky, K.S., et al., Use of biosynthetic human C-peptide in the measurement of insulin secretion rates in normal volunteers and type I diabetic patients. J Clin Invest, 1986. 77(1): p. 98-105.

52. Einstein, A., Elementary theory of the Brownian motion. Zeitschrift Fur Elektrochemie Und Angewandte Physikalische Chemie, 1908. 14: p. 235-239.

53. Gudbjornsdottir, S., et al., Direct measurements of the permeability surface area for insulin and glucose in human skeletal muscle. J Clin Endocrinol Metab, 2003. 88(10): p. 4559-64.

54. Sjostrand, M., A. Holmang, and P. Lonnroth, Measurement of interstitial insulin in human muscle. Am J Physiol, 1999. 276(1 Pt 1): p. E151-4.

55. Sjostrand, M., et al., Estimations of muscle interstitial insulin, glucose, and lactate in type 2 diabetic subjects. Am J Physiol Endocrinol Metab, 2000. 279(5): p. E1097-103.

56. Sjostrand, M., et al., Delayed transcapillary delivery of insulin to muscle interstitial fluid after oral glucose load in obese subjects. Diabetes, 2005. 54(1): p. 152-7.

57. Rabkin, R., M.P. Ryan, and W.C. Duckworth, The renal metabolism of insulin. Diabetologia, 1984. 27(3): p. 351-7.

58. Maack, T., et al., Renal filtration, transport, and metabolism of low-molecular-weight proteins: a review. Kidney Int, 1979. 16(3): p. 251-70.

59. Coulthard, M.G., Maturation of glomerular filtration in preterm and mature babies. Early Hum Dev, 1985. 11(3-4): p. 281-92.

60. Hann, C.E., et al., Integral-based parameter identification for long-term dynamic verification of a glucose-insulin system model. Comput Methods Programs Biomed, 2005. 77(3): p. 259270.

61. Le Compte, A.J., et al., Blood glucose prediction using stochastic modeling in neonatal intensive care. IEEE Trans Biomed Eng, 2010. 57(3): p. 509-18.

62. Bauer, K., et al., Body composition, nutrition, and fluid balance during the first two weeks of life in preterm neonates weighing less than 1500 grams. J Pediatr, 1991. 118(4 Pt 1): p. 61520.

63. Ekblad, H., et al., Extracellular volume in preterm infants: influence of gestational age and colloids. Early Hum Dev, 1991. 27(1-2): p. 1-7.

64. Heimler, R., et al., Relationship between Nutrition, Weight Change, and Fluid Compartments in Preterm Infants during the 1st Week of Life. Journal of Pediatrics, 1993. 122(1): p. 110114.

65. Modi, N., et al., Postnatal weight loss and contraction of the extracellular compartment is triggered by atrial natriuretic peptide. Early Hum Dev, 2000. 59(3): p. 201-8. 
66. Shaffer, S.G., et al., Extracellular fluid volume changes in very low birth weight infants during first 2 postnatal months. J Pediatr, 1987. 111(1): p. 124-8.

67. Shaffer, S.G. and V.M. Meade, Sodium balance and extracellular volume regulation in very low birth weight infants. J Pediatr, 1989. 115(2): p. 285-90.

68. Cassady, G., Plasma volume studies in low birth weight infants. Pediatrics, 1966. 38(6): p. 1020-7.

69. Leipala, J.A., et al., Blood volume assessment with hemoglobin subtype analysis in preterm infants. Biol Neonate, 2003. 84(1): p. 41-4.

70. Usher, R. and J. Lind, Blood Volume of the Newborn Premature Infant. Acta Paediatr Scand, 1965. 54: p. 419-31.

71. Lotz, T.F., et al., Monte Carlo analysis of a new model-based method for insulin sensitivity testing. Comput Methods Programs Biomed, 2008. 89(3): p. 215-25.

72. Pretty, C.G., et al., Interstitial insulin kinetic parameters for a 2-compartment insulin model with saturable clearance. Computer methods and programs in biomedicine, 2014. 114(3): p. e39-45.

73. Dickson, J.L., et al., Performance and Safety of STAR Glycaemic Control in Neonatal Intensive Care: Further Clinical Results Including Pilot Results from New Protocol Implementation, in 19th IFAC World Congress2014: Cape Town, South Africa.

74. Dickson, J.L., et al., Development and optimisation of stochastic targeted (STAR) glycaemic control for pre-term infants in neonatal intensive care. Biomedical Signal Processing and Control, 2013. 8(2): p. 215-221.

75. Le Compte, A.J., et al., Pilot study of a model-based approach to blood glucose control in very-low-birthweight neonates. BMC Pediatr, 2012. 12: p. 117.

76. Le Compte, A., et al. Blood glucose modelling and control for pre-term infants. in EPSM-ABEC 2008: Innovations in Patient Care. 2008. Christchurch, New Zealand.

77. Le Compte, A.J., et al., Blood Glucose Controller for Neonatal Intensive Care: Virtual Trials Development and First Clinical Trials. Journal of Diabetes Science and Technology, 2009. 3(5): p. 1066-1081.

78. Holliday, M.A., Metabolic rate and organ size during growth from infancy to maturity and during late gastation and early infancy. Pediatrics, 1971. 47(1): p. Suppl 2:169+.

79. Pretty, C.G., Analysis, classification and management of insulin sensitivity variability in a glucose-insulin system model for critical illness, in Mechanical Engineering2012, University of Canterbury: Christchurch, New Zealand. p. 169.

80. Cowett, R.M., W. Oh, and R. Schwartz, Persistent glucose production during glucose infusion in the neonate. J Clin Invest, 1983. 71(3): p. 467-75.

81. Lotz, T.F., et al. A fully identifiable physiological model of insulin kinetics for clinical applications. in 12th International Conf on Biomedical Engineering (ICBME). 2005. Singapore.

82. Polonsky, K.S., et al., Quantitative study of insulin secretion and clearance in normal and obese subjects. J Clin Invest, 1988. 81(2): p. 435-41. 